EPJ manuscript No.

(will be inserted by the editor)

\title{
Improving Multiparton Monte Carlo Tools in Hadronic Collisions ${ }^{\star}$
}

\author{
Fulvio Piccinini ${ }^{1}$ \\ INFN - Sezione di Pavia and Dipartimento di Fisica Nucleare e Teorica, via Bassi 6, 27100 Pavia, Italy \\ preprint numbers: CERN-TH/2003-267, FNT/T-2003/12
}

\begin{abstract}
Recent work on leading order multiparton calculations for hadronic collisions is reviewed, with special emphasis on the ALPGEN event generator. Some problems connected with the interface of the partonic events generated via matrix elements with the showering are addressed.
\end{abstract}

PACS. 12.38.Bx Perturbative calculations - 13.85.Hd Inelastic scattering: many-particle final states

\section{Introduction}

In high energy hadronic collisions multijet final states characterize a large class of important phenomena both within and beyond the SM. These multiparticle final states can originate from hard QCD radiation processes, decay of SM massive particles ( $W, Z$, top quark, Higgs boson), decay of heavy supersymmetric or more exotic particles. In general there are two different approaches to simulate multijet final states: the first one consists in generating the simplest possible final state by means of matrix elements and producing additional jets by parton showering (HERWIG [1, ISAJET 2], PYTHIA 3]). This procedure works well in the soft/collinear regions but fails to describe configurations with several widely separated jets. A complementary strategy is to generate high-multiplicity partonic final states by means of exact matrix elements and eventually pass the generated events to further showering. Even if it is a leading-order (LO) approach, this procedure can become very difficult because of the complexity of the matrix element calculations with many external legs and of the phase-space integration. Recently there has been extensive activity in developing several parton-level Monte Carlo (MC) event generators, such as ACERMC 4, ALPGEN 5], AMEGIC++ 6, CompHEP 7], GRACE 8],

HELAC/PHEGAS/JETI 9], MADEVENT [10. In this contribution the state of the art of the ALPGEN generator is reviewed, paying attention to the latest improvements. The general problem of interfacing a LO partonic event generator with the parton shower is addressed, reviewing some recent work on the subject.

\section{The ALPGEN event generator}

The ALPGEN library is a collection of MC codes dedicated to many processes relevant to high energy hadron-hadron

* Based on work done in collaboration with M.L. Mangano, M. Moretti, R. Pittau and A.D. Polosa. collisions. The calculations are based on partonic events generated by means of exact LO matrix elements, obtained with the ALPHA algorithm [11 for assigned kinematics, spin, flavour and colour configurations. The generated unweighted events, stored according to the Les Houches Accord \#1 12] format, are ready for the HERWIG/PYTHIA evolution from partons to hadrons. Up to now the available final states in the ALPGEN package are:

- $W+N$ jets, $Z / \gamma^{*}+N$ jets, $N \leq 6$,

- $W Q \bar{Q}+N$ jets, $Z / \gamma^{*} Q \bar{Q}+N$ jets $(Q=c, b, t), N \leq 4$,

$-W+c+N$ jets, $N \leq 5$,

$-n W+m Z+p$ Higgs $+N$ jets, $n+m+p \leq 8, N \leq 3$,

$-m \gamma+N$ jets, $N+m \leq 8$ and $N \leq 6$,

$-Q \bar{Q}+N$ jets, $(Q=c, b, t), N \leq 6$,

- $Q \bar{Q} Q^{\prime} \bar{Q}^{\prime}+N$ jets, $\left(Q, Q^{\prime}=b, \bar{t}\right), N \leq 4$,

$-N$ jets, $N \leq 6$,

$-Q \bar{Q} H+N$ jets, $(Q=b, t), N \leq 4$.

The limitations in the maximum number of jets is only due to the setting of internal parameters in the ALPHA code, which could be changed to accomodate a larger number of final-state particles. While in the first version of ALPGEN the top quarks were considered as real particles, they are now (v1.3, in the $Q \bar{Q}$ and $Q \bar{Q} H$ processes) allowed to decay in the three final state fermions $\left(t \rightarrow W b \rightarrow b f \bar{f}^{\prime}\right)$ with exact matrix element, thus retaining all the spin correlations among the top decay products. The decay is calculated in the approximation of on-shell top quark and $W$ boson, in order to avoid the inclusion of non-resonant diagrams while preserving the gauge invariance of the calculations. The same strategy has been implemented for the decay of the gauge boson in the vbjets code, the ALPGEN directory dedicated to multiboson plus jets production, where the matrix element for the vector boson decay into a fermionic pair has been introduced in the zero width approximation. The generation of multiboson final states requires a careful treatment of the widths in the propagators of the unstable particles, because they generally break gauge invariance, giving rise to a bad high 
energy behaviour of the cross sections 13. The strategy adopted in ALPGEN is to calculate the matrix element with the bosonic widths set to zero, removing the events containing a vector boson with a propagator mass $M_{0}$ such that $\left|M_{0}^{2}-M^{2}\right|<s_{0}$, with

$$
\int_{-\infty}^{M^{2}-s_{0}} d s \frac{1}{\left(s-M^{2}\right)^{2}}=\int_{-\infty}^{M^{2}} d s \frac{1}{\left(s-M^{2}\right)^{2}+\Gamma^{2} M^{2}}
$$

and

$$
\left|M_{0}-M\right| \leq \frac{\Gamma}{\pi}
$$

For all other processes that do not involve the presence of several gauge bosons, the fixed-widths prescription is used. The Higgs bosons are treated as stable particles. Their decay to fermion pairs or to four fermions will be implemented in a future release of the programme.

\section{Matching partonic event generators to parton shower}

In order to simulate the real hadronic final states, the partonic events have to be passed through a MC parton shower. However, in this interface there is some ambiguity in the cuts implemented at the partonic level. They are unphysical, in the sense that the final jet cross-section should be independent of their choice, provided that they are not harder than the cuts applied to the real jets. However, starting with looser partonic cuts increases the probability of obtaining $n$ jets from $n+m$ partons after parton showering (giving rise to the double-counting problem), as can be seen in fig. [1 which shows the jet rates with the constraint $E_{T}^{\text {jet }}>E_{T}^{\text {cut }}$ for the hardest jet in $W+3$ jets events at Tevatron, versus the parton separation $\Delta R_{\text {part }}$ imposed at the level of ME generation. The jets are reconstructed with the cone algorithm and the cross sections are normalized to the result obtained with $\Delta R_{\text {part }}=0.7$. The increasing ratio for smaller $\Delta R_{\text {part }}$ is due to both the collinear divergence of the matrix element for $\Delta R_{\text {part }} \rightarrow 0$ and the increasing double counting for smaller $\Delta R_{\text {part }}$. A first approximation to the solution of the problem could consist in requiring a jet matching for every parton [14. With this recipe the shapes of the $W+3$ jets example above become flatter, as diplayed in fig. 2. but still showing a residual dependence on the parameter $\Delta R_{\text {part }}$. The general problem of matching multiparton matrix element calculations with parton showers has been extensively studied in the literature and for $e^{+} e^{-}$collisions a solution (CKKW) has been proposed and tested on LEP data 15], which avoids double counting and shifts the dependence on the resolution parameter beyond next-to-leading logarithmic (NLL) accuracy. The method consists in separating arbitrarily the phase-space regions covered by matrix element and parton shower, and use, for all parton multiplicities, vetoed parton showers together with reweighted tree-level matrix elements by means of suitable Sudakov form-factor combinations. The necessary steps for the implementation of the procedure can be summarized as follows:

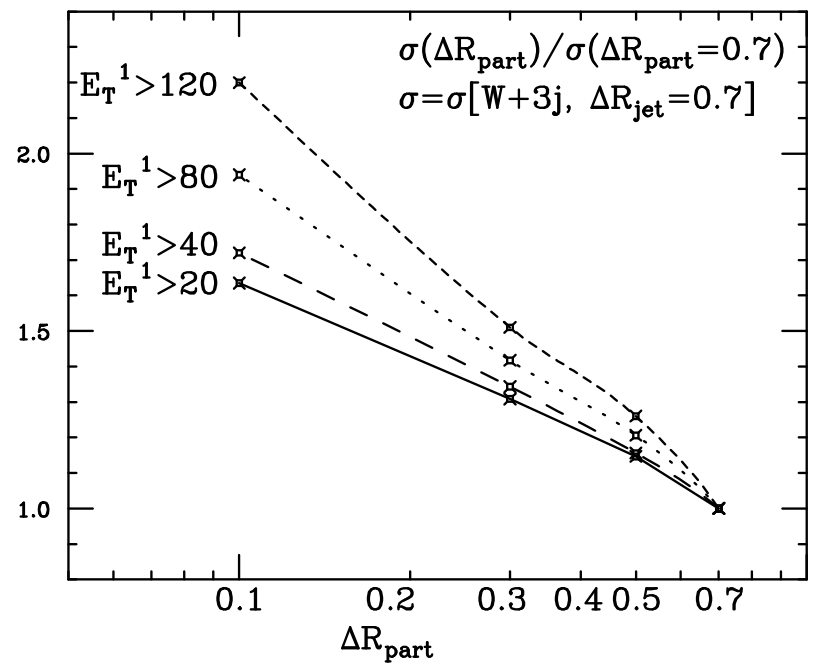

Fig. 1. The rate for $p \bar{p} \rightarrow W+3$ jets at Tevatron as a function of the partonic separation cut $\Delta R_{\text {part }}$ normalized to the cross section for $\Delta R_{\text {part }}=0.7$.

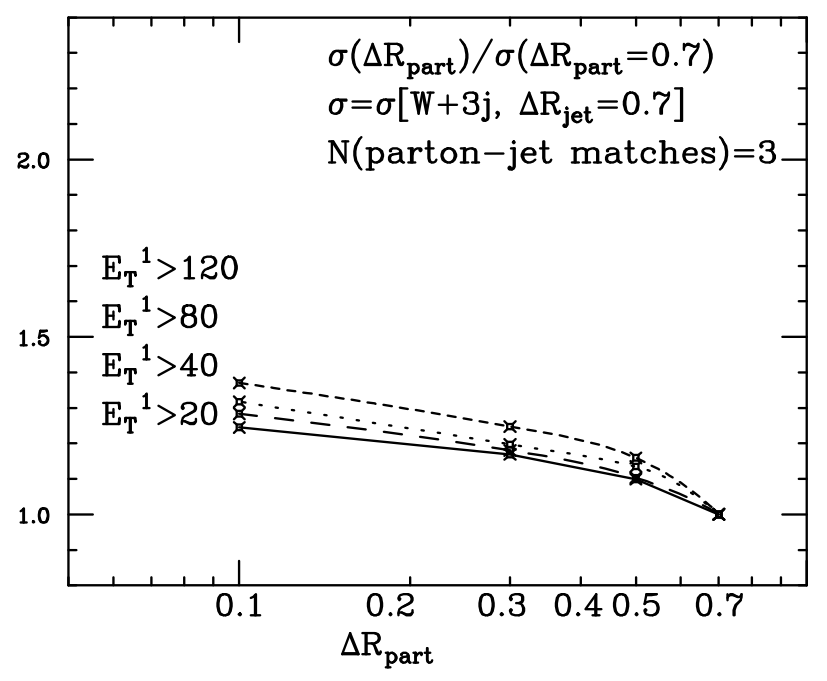

Fig. 2. The same as in fig. 1 requiring a jet matching for every parton 14 .

- select the jet multiplicity $n$ according to the jet rates obtained with matrix elements with resolution $y_{i j}>$ $y_{\text {cut }}$, defined according to the $k_{T}$-algorithm [16] $\left(y_{i j}=\right.$ $\left.2 \min \left(E_{i}^{2}, E_{j}^{2}\right) / \hat{s}\left(1-\cos \vartheta_{i j}\right)\right)$;

- generate $n$ parton momenta according to the matrix element with fixed $\alpha_{s}\left(y_{c u t}\right)$ and reweight the event with the probability of no further branching by means of Sudakov form factors;

- build a "PS history" by clustering the partons to determine the values at which $1,2, \ldots, n$ jets are resolved. In so doing a tree of branchings is constructed and the nodal scales characteristic of each branching are used to reweight the event with running $\alpha_{s}$;

- apply a coupling constant reweighting factor $\alpha_{s}\left(y_{i}\right) /$ $\alpha_{s}\left(y_{\text {cut }}\right) \leq 1$ for every branching of the "PS history", where $y_{i}$ is the nodal scale; 
- after successful unweighting, use the $n$-parton kinematics as initial condition for the shower, vetoing all branchings such that $y_{i j}>y_{\text {cut }}$.

The extension of the procedure to hadronic collisions has been proposed in ref. [17. Recent detailed results of the implementation of the procedure with the programs HERWIG, PYTHIA and SHERPA have been presented in ref. 18. The implementation in ALPGEN is currently under investigation. Preliminary studies regarding the partonic steps with ALPGEN have been presented in ref. [19]. In the case of hadronic collisions there is a certain degree of arbitrariness, such as the choice of the Sudakov form factors, the choice of the scale of $\alpha_{s}$ (LO or NLO), the treatment of the highest-multiplicity matrix element, the choice of the clustering scheme, the use of flavour or colour information to define the tree and the related reweighting factors, the treatment of gauge bosons. All these uncertainties entail that a large degree of tuning on the data (possibly processdependent) will be needed, and further work remains to be done to find what the correct prescriptions are. The $\alpha_{s}$ reweighting of the partonic events could be important on its own, because it should effectively give, in a gaugeinvariant way, the bulk of the NLO QCD corrections. This could be tested in cases where multijet NLO calculations are available.

\section{Summary}

The MC simulation of hard multiparticle final states at hadronic colliders is a very important issue. Thanks to recent efforts by different groups, several multiparton event generators based on exact matrix elements are now available; they were thoroughly cross-checked during the MC workshops held at FNAL and CERN during 2003. These programs generate samples of unweighted events in a standardized format (the LesHouches Accord \# 1) which can be passed to the parton shower-based MC programs to go from the partons to the real final-state hadrons. The matching between a LO multijet event generator and the parton shower MC suffers from the serious problems of double-counting and dependence on the parton-level cuts. For the case of $e^{+} e^{-}$collisions the problem has been solved beyond NLL accuracy with the CKKW algorithm. This can be extended to hadronic collisions, but the proof is still pending. However, recently, there has been an intense activity in its implementation on existing $\mathrm{MC}$ event generators going through many subtleties involved in the CKKW algorithm for hadronic collisions.

The author wishes to thank the conveners of the Hard QCD Parallel Session for the invitation. M.L. Mangano and A.D. Polosa are gratefully aknowledged for many useful discussions and a careful reading of the manuscript.

\section{References}

1. G. Marchesini and B. R. Webber, Nucl. Phys. B 310 (1988) 461. G. Marchesini, et al., Comput. Phys. Commun.
67 (1992) 465. G. Corcella et al., JHEP 0101 (2001) 010 hep-ph/0011363.

2. F. E. Paige, S. D. Protopopescu, H. Baer and X. Tata, hep-ph/9810440

3. T. Sjöstrand, Comput. Phys. Commun. 82 (1994) 74; T. Sjöstrand, et al., Comput. Phys. Commun. 135 (2001) 238.

4. B.P. Kersevan and E. Richter-Wąs, hep-ph/0201302

5. M.L. Mangano et al., JHEP 07 (2003) 001.

The documentation and the codes can be found at http://mlm.home.cern.ch/mlm/alpgen/

6. F. Krauss, R. Kuhn and G. Soff, JHEP 0202 (2002) 044.

7. A. Pukhov et al., hep-ph/9908288

8. T. Ishikawa et al. [MINAMI-TATEYA group Coll.], KEK92-19; S. Tsuno et al., Comput. Phys. Commun. 151 (2003) 216.

9. P. D. Draggiotis, R. H. Kleiss and C. G. Papadopoulos, Eur. Phy. J. C 24 (2002) 447; C.G. Papadopoulos, talk given at 22 .

10. T. Stelzer and W.F. Long, Comput. Phys. Commun. 81 (1994) 357; F. Maltoni and T. Stelzer, JHEP 0302 (2003) 027.

11. F. Caravaglios and M. Moretti, Phys. Lett. B 358 (1995) 332; F. Caravaglios, M. L. Mangano, M. Moretti and R. Pittau, Nucl. Phys. B 539 (1999) 215.

12. E. Boos et al., hep-ph/0109068

13. M. Veltman, Physica 29 (1963) 186; U. Baur, J.A.M. Vermaseren and D. Zeppenfeld, Nucl. Phys. B 375 (1992) 3; Y. Kurihara, D. Perret-Gallix and Y. Shimizu, Phy. Lett. B 349 (1995) 367; U. Baur and D. Zeppenfeld, Phys. Rev. Lett. 75 (1995) 1002; R.G. Stuart, Phys. Lett. B 262 (1991) 113; hep-ph/9603351 hep-ph/9706431 hep-ph/9706550 A. Aeppli, G.J. van Oldenborgh and D. Wyler, Nucl. Phys. B 428 (1994) 126; C.G. Papadopoulos, Phys. Lett. B 352 (1995) 144; E.N. Argyres et al., Phys. Lett. B 358 (1995) 339; W. Beenakker et al., Nucl. Phys. B 500 (1997) 255; W. Beenakker, F.A. Berends and A.P. Chapovsky, Nucl. Phys. B 548 (1999) 3; W. Beenakker et al., hep-ph/0303105 G. López Castro et al., Mod. Phys. Lett. A40 (1991) 3679; A. Denner et al., Nucl. Phys. B 560 (1999) 33; E. Accomando, A. Ballestrero and E. Maina, Phys. Lett. B 479 (2000) 209.

14. M.L. Mangano, talk given at 20, 15 November 2002.

15. S. Catani et al., JHEP 0111 (2001) 063; L. Lönnblad, JHEP 0205 (2002) 046; R. Kuhn et al., hep-ph/0012025 F. Krauss, R. Kuhn and G. Soff, J. Phys. G 26 (2000) L11. 16. S. Catani, Y.L. Dokshitzer and B.R. Webber, Phys. Lett. B 285 (1992) 291; S. Catani, Y.L. Dokshitzer, M.H. Seymour and B.R. Webber, Nucl. Phys. B 406 (1993) 187.

17. F. Krauss, JHEP 0208 (2002) 015.

18. S. Mrenna, talks given at 202122 ; P. Richardson, talks given at 202122$]$; F. Krauss, talk given at [20]; A. Schälicke, talk given at 22$]$.

19. M.L. Mangano, talks given at 2022 .

20. Monte Carlo Tuning Working Group, FNAL, 29-30 April 2003; http://cepa.fnal.gov/CPD/MCTuning/

21. Workshop "Physics at TeV Colliders", Les Houches, 26 May - 6 June 2003; http://lappc-in39.in2p3.fr/conferences/LesHouches/ Houches2003/.

22. Workshop on "MC tools for the LHC", CERN, 7 July - 1 August 2003;

http://mlm.home.cern.ch/mlm/mcwshop03/mcwshop.html 